\title{
More than a feeling?-Overruling the preoperatively templated offset option leads to a minor offset increase in short stem total hip arthroplasty
}

\author{
Matthias Luger ${ }^{1,2}$ D $\cdot$ Christian Stadler ${ }^{1,2} \cdot$ Rainer Hochgatterer $^{1,2} \cdot$ Jakob Allerstorfer $^{1,2} \cdot$ Tobias Gotterbarm $^{1,2}$. \\ Antonio Klasan ${ }^{1,2}$
}

Received: 9 July 2021 / Accepted: 19 December 2021 / Published online: 7 January 2022

(c) The Author(s) 2022

\begin{abstract}
Purpose Short stems are increasingly used in total hip arthroplasty (THA) because of advantages in bone and soft tissue preservation and reconstruction of hip geometry. Digital templating is essential in determining the correct offset option and stem size in THA. However, the preoperative template sizes might be intraoperatively overruled.

Patients and methods We evaluated the effect of intraoperative overruling of the preoperatively templated offset option of a short curved stem on hip offset, leg length, implant positioning, and femoral canal fill index. The overruling was performed in case of intraoperative instability, telescoping, or both. A series of 1052 consecutive THAs with a cementless short curved stem and press-fit cup was retrospectively screened. One hundred patients with unilateral THA and a contralateral native and morphologically healthy hip as a reference met the inclusion criteria. Measurements were carried out on preoperative and 3 months anterior-posterior postoperative radiographs. Patients were divided according to the overruling by offset option or stem size.

Results Hip offset was increased in all groups, but only with significant increase if an offset option +1 was used intraoperatively $(p=0.025)$. LLD was restored without significance in all groups $(p=0.323 ; p=0.157)$.

Conclusion Intraoperative overruling of the preoperative digital template in cementless short stem total hip arthroplasty results in an increase of hip offset compared to a contralateral healthy hip. However, the increase is marginal and clearly under $5 \mathrm{~mm}$ compared to the contralateral healthy hip.
\end{abstract}

Keywords Short stem $\cdot$ Total hip arthroplasty $\cdot$ Digital templating $\cdot$ Femoral offset $\cdot$ Hip offset $\cdot$ Leg length

Matthias Luger

Matthias.luger@kepleruniklinikum.at

Christian Stadler

christian.stadler@kepleruniklinikum.at

Rainer Hochgatterer

Rainer.hochgatterer@kepleruniklinikum.at

Jakob Allerstorfer

Jakob.allerstorfer@kepleruniklinikum.at

Tobias Gotterbarm

Tobias.gotterbarm@kepleruniklinikum.at

Antonio Klasan

Klasan.antonio@me.com

1 Department for Orthopedics and Traumatology, Kepler University Hospital GmbH, Krankenhausstrasse 9, 4020 Linz, Austria

2 Johannes Kepler University Linz, Altenberger Strasse 69, 4040 Linz, Austria

\section{Introduction}

Short stem total hip arthroplasty (THA) is increasingly performed in recent years $[1,2]$. Short stems have the advantage of superior preservation of the proximal bone stock [3], facilitate a more soft tissue conserving implantation in minimally invasive approaches [2, 4], and restore the hip geometry superiorly [5]. Conventional straight stems show excellent long-term outcomes [6], but have a limited ability to restore the femoral offset (FO) due to their straight geometry [5]. Besides leg length (LL), FO is an essential parameter for postoperative clinical functional outcome, dislocation rate, wear, and revision rate [5]. Restoration of the native FO increases postoperative range of motion, abductor muscle function, and decreases polyethylene wear [5, 7]. Several studies even suggest a beneficial effect of an increased FO on abductor muscle force and joint reaction $[8,9]$. 
A successful postoperative outcome after total hip arthroplasty relies on restoring the biomechanics of the hip as well as selecting the appropriate implant size $[10,11]$. The position of short stem implants is more variable and also depends on the femoral resection level and the anatomy of the femoral neck [12]. Therefore, preoperative templating is found as an integral part of selecting the correct implant size intraoperatively in order to avoid complications [13]. Undersizing can lead to component loosening, while oversizing can lead to intraoperative fractures $[14,15]$.

Preoperative templating in short stem THA is essential for determining the correct implant sizes in order to reconstruct the hip offset and restore the leg length adequately. While accuracy in digital templating for short stem THA is described as reliable compared to conventional straight stems [16], other studies suggest a lower rate of accuracy for short stems [17]. However, accurate digital templating is heavily dependent on a standardized and correctly applied imaging technique as well as the correct calibration of the digitalized radiography $[18,19]$. In certain cases, surgeons may have to overrule the preoperative digital template in order to achieve sufficient joint stability. The effects on hip offset, leg length difference, and implant positioning in these cases of overruling preoperative templates in short stem THA are unknown. We therefore conducted this study to evaluate the effect on hip geometry, leg length, and implant positioning in THA with a cementless curved short stem with four different offset options.

\section{Methods}

\section{Patients}

This retrospective radiological comparative study includes patients of a consecutive series of THAs with the same cementless curved short stem (Fitmore ${ }^{\circledR}$ stem, ZimmerBiomet, Warsaw, IN, USA) and bi-hemispherical press-fit acetabular cup (Allofit ${ }^{\circledR} /$-S, ZimmerBiomet, Warsaw, IN, USA) performed via a minimally invasive supine anterolateral approach. Fitmore ${ }^{\circledR}$ hip stem is a titanium alloy stem (Ti Al6V4) that has a porolock Ti-VPS coating in the proximal part to enhance bone ingrowth and is available in four different neck angle options $\left(127^{\circ}, 129^{\circ}, 137^{\circ}, 140^{\circ}\right)$ and 14 different sizes (size 1-14) for each offset option [2]. A cementless titanium press-fit cup with or without screws (Allofit ${ }^{\circledR} /$-S, ZimmerBiomet, Warsaw, IN, USA) was used in all patients.

A consecutive series of 1052 hips in 982 patients with index surgery between 2014 and 2019 were screened for inclusion and the medical records until 90 days postoperative were evaluated. Firstly, all patients were screened for existence of a preoperative digital templating. In 63 cases there was no preoperative template available. In a second step preoperative X-rays (both hips in comparison, anterior-posterior view, standing upright) of these 989 cases were screened for unilateral THA. Diagnoses for inclusion were primary osteoarthritis, avascular necrosis of the femoral head, or mild dysplasia of the hip (Crowe I) [20]. Exclusion criteria were defined as bilateral hip disease (Kellgren Lawrence $>$ grade 1) [21], a history of prior hip surgery, previous trauma, postoperative complication, reoperation or revision for any reason, as well as missing pre- or postoperative radiographs. In total 100 patients met the inclusion criteria. Overruling the preoperative template was defined in different groups, Table 1. The offset of the Fitmore ${ }^{\circledR}$ hip stem is primarily affected by the offset option itself and secondly by the stem, as an increasing stem size results also in an increased horizontal offset and vice versa.

Radiographic measurements were performed on preoperative and 3-month postoperative low-centered anteroposterior (AP) radiographs of the pelvis in both groups.

Table 1 Description of the different overruled offset options and stem sizes

\begin{tabular}{|c|c|c|}
\hline & Description & $\begin{array}{l}\text { Number of } \\
\text { cases }(n)\end{array}$ \\
\hline Total & & 100 \\
\hline Offset 0 & Offset option intraoperatively used as templated & 67 \\
\hline Offset +1 & One offset option higher than templated used intraoperatively & 21 \\
\hline Offset -1 & One offset option lower than templated used intraoperatively & 11 \\
\hline Offset -2 & Two offset options lower than templated used intraoperatively & 1 \\
\hline Total & & 100 \\
\hline Offset $0 /$ Size 0 & Offset option as templated used intraoperatively; Stem size used intraoperatively as templated & 27 \\
\hline Offset $0 /$ Size $>+1$ & $\begin{array}{l}\text { Offset option as templated used intraoperatively; Stem size bigger } 1 \text { size or more used intraoperatively as } \\
\text { templated }\end{array}$ & 6 \\
\hline Offset $0 /$ Size $>-1$ & $\begin{array}{l}\text { Offset option as templated used intraoperatively; Stem size smaller } 1 \text { size or more used intraoperatively as } \\
\text { templated }\end{array}$ & 34 \\
\hline Offset \pm 1 option & Other offset option as templated used intraoperatively & 33 \\
\hline
\end{tabular}


Preoperative age at operation, gender, body mass index (BMI), and laterality were recorded. The patient demographics are shown in Table 2 and were evaluated in two different groups: offset option intraoperatively used as templated (Group A) and any deviation in offset option implanted intraoperatively (Group B).

This study was approved by the institutional review board (EK-No.: 1239/2019). Due to the retrospective study design with evaluation of pre-existing medical records, an informed consent was not required. All procedures performed in studies involving human participants were in accordance with the ethical standards of the institutional and/or national research committee and with the $1964 \mathrm{Hel}-$ sinki declaration and its later amendments or comparable ethical standards.

Table 2 Patient demographics, (Mean and SD), and Testing

\begin{tabular}{lllc}
\hline Variable & Group A & Group B & $p$ value \\
\hline Number of Hips & 67 & 33 & - \\
Side (L:R) & $31: 36$ & $15: 18$ & 1.000 \\
Gender (F:M) & $44: 23$ & $17: 16$ & 0.196 \\
Age (years) & $55.6 \pm 10.5$ & $56.8 \pm 11.5$ & 0.772 \\
& $(35-77.1)$ & $(27-75.2)$ & \\
BMI at surgery & $27.1 \pm 4.1(19.7-$ & $29.3 \pm 5.9(20.5-$ & 0.078 \\
& $36.5)$ & $47.3)$ & \\
\hline
\end{tabular}

$S D$ standard deviation, $F$ female, $M$ male, $L$ left, $R$ right, $B M I$ body mass index $\left(\mathrm{kg} / \mathrm{m}^{2}\right)$

\section{Digital templating}

Digital templating was carried out with mediCAD ${ }^{\circledR}$ version 5.1 (Hectec GmbH, Altdorf, Germany) in a standardized manner. Scaling was performed using a $25-\mathrm{mm}$ metallic radiopaque ball as the reference, placed between the legs of the patient, aiming for the level of the hip in the frontal plane. The scaling was performed automatically by the software. Then, the center of rotation, the proximal femoral shaft axis, and the leg length discrepancy were determined. After that the correct size and position of the acetabular component were determined. Next the size of the femoral component was templated beginning with the correct offset option. The aim in templating a Fitmore hip stem is to restore the anatomical offset by confirming that the medial curve of the stem follows closely to the inner line of the cortex in the calcar region when the stem is in axis with the femoral canal. After choosing the correct offset option, the appropriate stem size is selected. The appropriate stem size is selected by choosing the stem which fills the intramedullary canal entirely. Figure 1 shows an example for digital templating as carried out in this study. In the presented study, the operating surgeon performed the preoperative digital template by a standardized templating procedure as explained above. However, the neck length was not templated in every case and was therefore not included for statistical analysis. All preoperative templates included cup size, stem offset, and stem size.

Overruling of the digital template leading to an increased offset was done by the operating surgeon in case of
Fig. 1 Preoperative digital template on an anterior-posterior radiograph of the pelvis

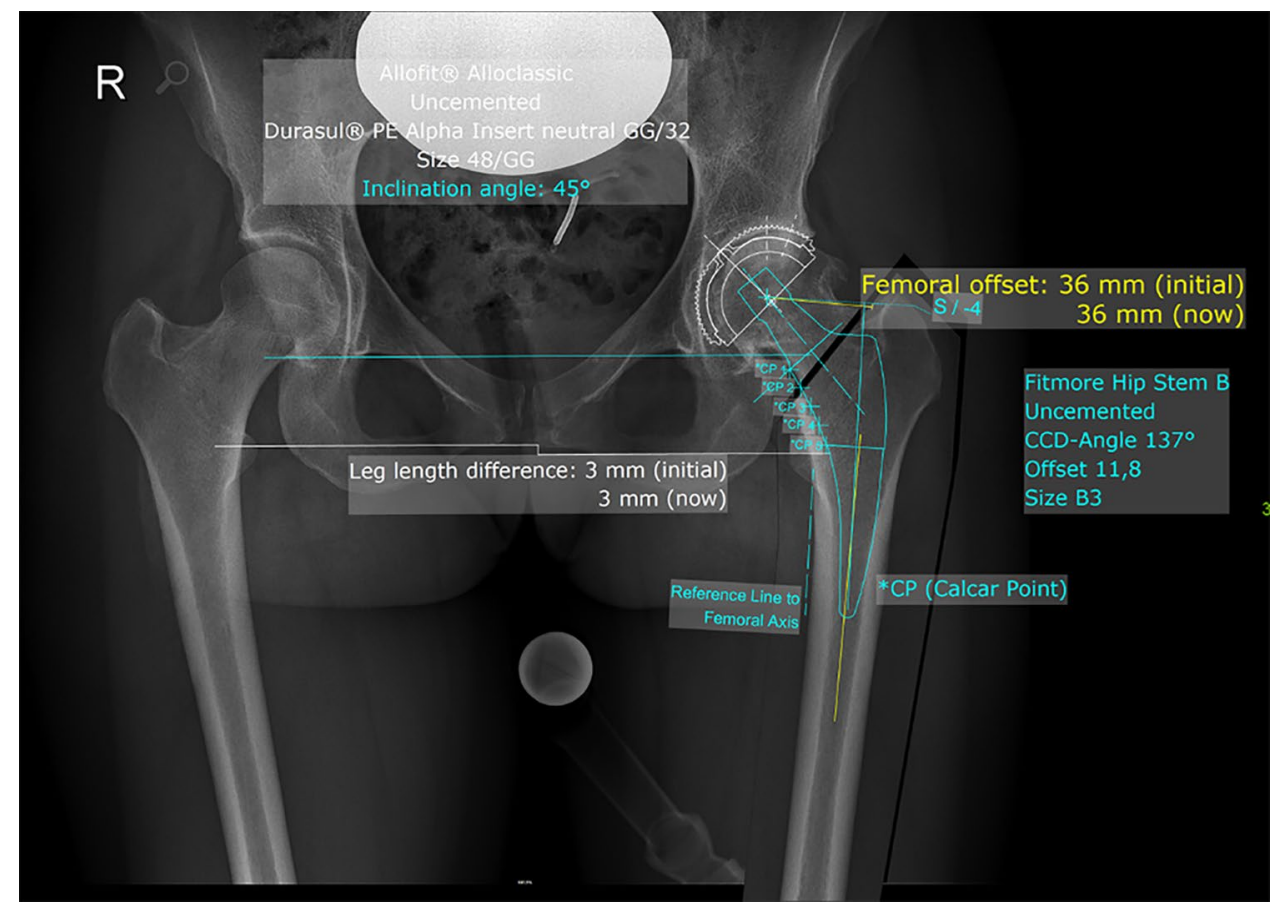


intraoperative necessity due to instability, "telescoping," or both after trial reduction. A higher offset option was chosen if stability could not be achieved by a higher head size offset. In case of increased of leg length difference discrepancy and instability or in case of impending leg length difference by using a higher head size, a higher offset option was chosen. This decision was performed at the discretion of the surgeon, based on intraoperative judgment and experience.

\section{Surgical technique and treatment protocol}

Surgical procedures were carried out at the author's institution by surgeons with different levels of experience, including 11 consultants and 7 residents. All consultants perform more than 50, all senior consultants more than 100 arthroplasties per year. Resident surgeries were done under the guidance of a consultant. In all cases a minimally invasive anterolateral Watson-Jones approach in supine position on a standard operating table under laminar air flow was performed. Extremity preparation was performed with threefold antiseptic scrub with alcohol disinfectant. Draping with a sterile adhesive surgical iodine film was used. The skin incision was centered over the greater trochanter. An incision at the border between the Tensor fasciae latae and the Tractus iliotibialis was performed. Then the Watson-Jones interval between Tensor fasciae latae and Gluteus medius was bluntly dissected. A capsulectomy was performed in every case. Fluoroscopy was routinely used with the definitive cup and trial stem in situ. The standardized peri- and postoperative protocol was identical in all cases, including single-shot antibiotics (Cefuroxime $1.5 \mathrm{~g}$ i.v. directly preoperative), weight bearing as tolerated from the first postoperative day on, Indomethacin $75 \mathrm{mg}$ daily for the prevention of heterotopic ossification on days 1 to 4 postoperatively and $40 \mathrm{mg}$ low-molecular weight heparin, or Rivaroxaban $10 \mathrm{mg}$ for 28 days postoperatively as venous thromboembolic event prophylaxis.

\section{Radiographic evaluation}

Radiographic measurement was performed on preoperative and 3-month postoperative digital low-centered AP radiographs of the pelvis [22]. Measurement was conducted independently by two reviewers (M.L., J.A.), who were not involved in the index surgery. Radiographs were taken with the patient in standing position and with both legs in $15^{\circ}$ internal rotation and the central beam was directed on the symphysis pubis [23]. In order to achieve an accurate measurement of the hip anatomy a double coordinate system was applied on both the preoperative and the postoperative images $[1,24]$. Radiographic analysis was also performed using MediCAD ${ }^{\circledR}$ Software V5.1. The hip center of rotation (COR) was defined using a circle tool determining the diameter of the femoral head and its center [25]. The femoral offset (FO) was determined as the perpendicular distance between the COR and the proximal femoral shaft axis (FSA) $[22,25]$. Acetabular offset (AO) was measured as the perpendicular distance between the COR and line $\mathrm{T}$, with $\mathrm{T}$ being the perpendicular line on the trans-teardrop line (TT) through the ipsilateral teardrop figure [22]. Hip offset (HO) was calculated as the sum of FO and AO [22]. The vertical position of the COR was measured as the perpendicular distance to line TT [26]. Radiographic leg length discrepancy (LLD) was measured as the perpendicular distance between line TT and the middle of the lesser trochanter (LT) [23]. Centrum-Collum-Diaphyseal (CCD) angle was determined according to M. E. Müller on the affected hip [27]. To characterize the anatomical shape of the proximal femur and the thickness of cortical bone, the canal to calcar isthmus ratio and the cortical index (CI) according to Dorr et al. [28] were determined. A high CI indicates a thick cortical bone [28]. Additionally the canal flare according to Noble et al. [29] was determined. On preoperative X-rays FO, AO, HO, LLD, and vertical position of the COR were measured bilaterally, while CCD angle, CI, Canal Flare Index, and Canal to Calcar Ratio were measured unilaterally on the affected hip. Complete preoperative measurements are also shown in Fig. 2.

On postoperative X-rays FO, AO, HO, LLD, and vertical position of the COR were measured bilaterally. Complete postoperative measurements are also shown in Fig. 3.

Intra- and interobserver reliabilities were calculated for 15 randomly selected cases for each group. Intraclass correlation coefficients (ICC) were used with a two-way random effects model for absolute agreement. Repeated measurements for intraobserver reliability were performed at day 1 and day 14 in a blinded fashion.

\section{Statistics}

Descriptive statistical analysis was conducted for age, gender, body mass index (BMI), and laterality. A Shapiro-Wilk test was performed for testing normal distribution. As not all variables were normally distributed non-parametric testing was performed. For patient demographics a Fisher's exact test was performed on categorical variables (gender and laterality). A Wilcoxon-Mann-Whitney $U$ test was performed on continuous variables (age and BMI). For statistical analysis of pre- and postoperative radiographic measurements, a non-parametric Kruskal-Wallis test was performed. Power analysis was not performed due to the observed statistical significance [30]. Therefore, the null hypothesis was to find out that overruling the preoperative digital template in total hip arthroplasty does not affect hip offset compared to the contralateral healthy hip. Statistical analysis was calculated with SPSS version 26 (IBM SPSS statistics, Chicago, 
Fig. 2 Preoperative measurements: Both sides: $F O$ femoral offset, $A O$ acetabular offset, $C C D$ angle vertical position of the center of rotation (COR), Leg length difference (LLD); Affected hip: Centrum-CollumDiaphyseal Angle, $C I$ cortical index, Canal Flare Index Canal to Calcar ratio

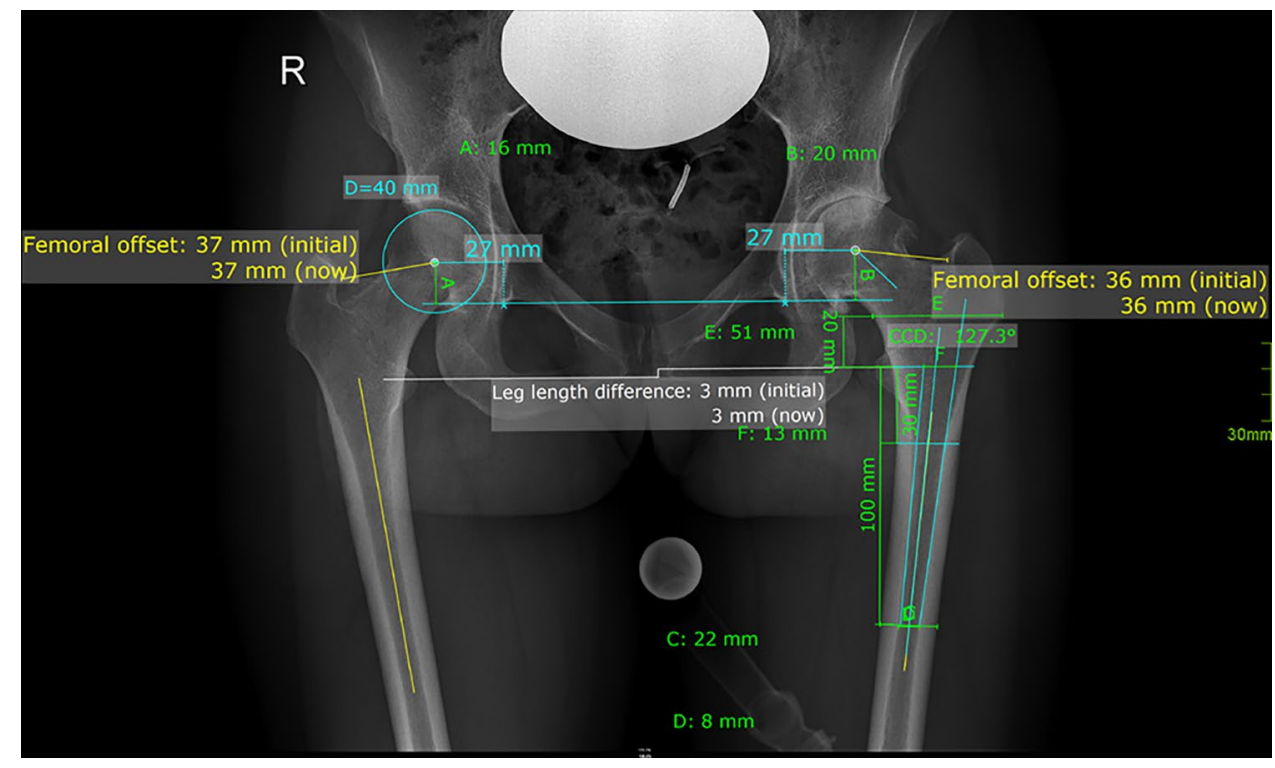

Fig. 3 Both sides: $F O$ femoral offset, $A O$ acetabular offset, $C O R$ vertical position of the center of rotation, $L L D$ Leg length difference

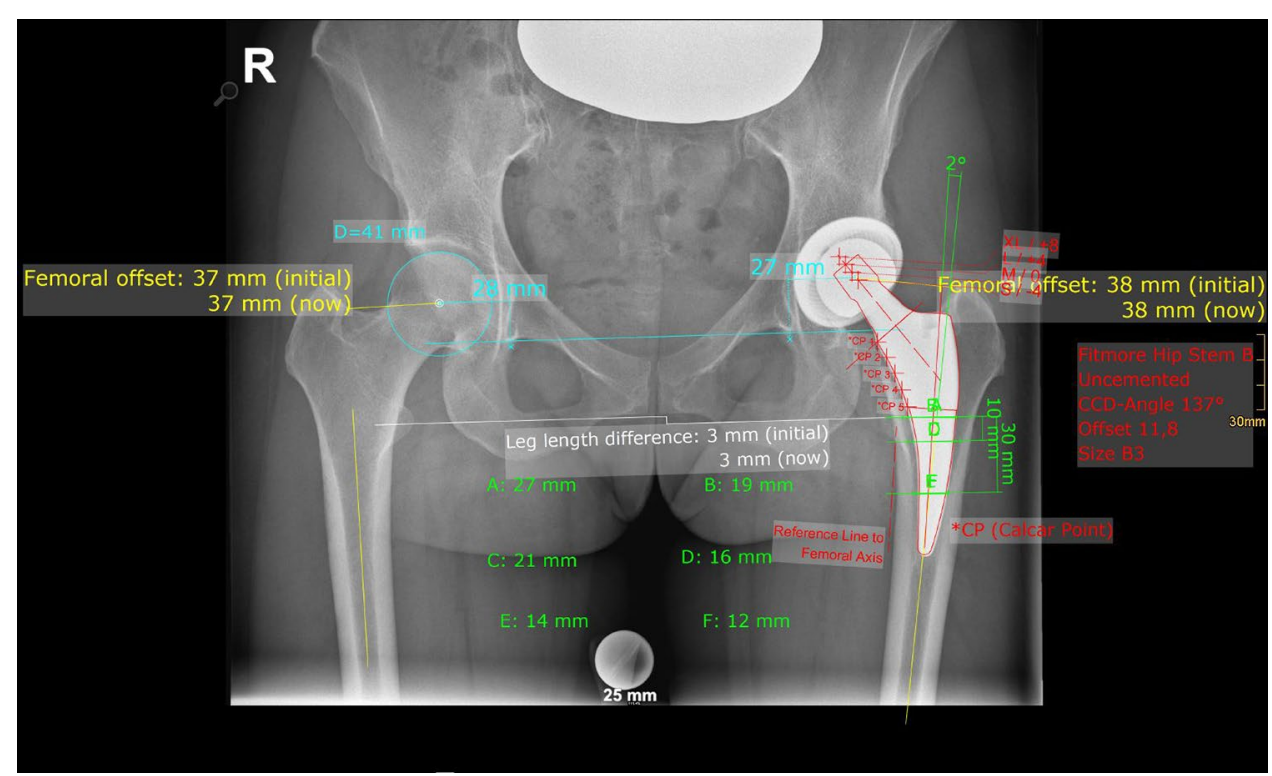

IL, USA). A $p$ value $<0.05$ was considered as statistically significant.

\section{Results}

The interobserver and intraobserver correlation coefficient for radiographic measurements showed satisfying results (range, 0.961 [95\% CI, 0.853-0.989] to 0.998 [95\% CI, 0.986-0.999]). Average age at operation, gender, BMI, and laterality were evenly distributed and did not show any statistical significance as shown in Table 3. Difference in HO, AO, FO, LLD, and vertical position of the COR did not show any statistical difference in both groups as shown in Table 3. Only the CCD angle showed statistically significant differences, Table 3.
Anatomical shape of the affected side did not show any significance in testing of the Cortical Index, Canal Flare Index, and Canal to Calcar ratio.

Table 4 shows the postoperative measurements in detail. Postoperative HO only increased if an offset option +1 option was used $(p=0.025)$. Apart from group "Offset -2 " AO was reduced in all groups, Table 4. FO was increased in all groups, Table 4. The vertical center of rotation was cranialized significantly in all groups, Table 4. The LLD did not show significant differences between the groups, Table 4 . 
Table 3 Preoperative radiographic measurements

\begin{tabular}{lrcclc}
\hline $\begin{array}{l}\text { Preoperative discrepancy between } \\
\text { arthritic and healthy hip }\end{array}$ & \multicolumn{1}{l}{ Offset 0} & Offset -1 & Offset +1 & Offset -2 & $p$ value \\
\hline Hip offset $(\mathrm{mm})$ & $1.5 \pm 3.8$ & $0 \pm 4.7$ & $0.9 \pm 4.7$ & -3 & 0.286 \\
Acetabular offset (mm) & $0.5 \pm 2.5$ & $2.5 \pm 3.2$ & $0.7 \pm 1.7$ & 1.00 & 0.298 \\
Femoral offset (mm) & $2 \pm 3.9$ & $2.5 \pm 5$ & $1.6 \pm 4.5$ & -2 & 0.622 \\
Leg length difference (mm) & $-4.1 \pm 4.4$ & $-3.4 \pm 6.7$ & $-2.7 \pm 5$ & 4.00 & 0.323 \\
Vertical position of the COR (mm) & $2.9 \pm 3.9$ & $0.6 \pm 4.2$ & $2.5 \pm 2.8$ & -2.00 & 0.119 \\
CCD angle $\left(^{\circ}\right)$ & $131.9 \pm 6.7$ & $126.9 \pm 5.5$ & $131.6 \pm 5.8$ & 119.8 & $\mathbf{0 . 0 4 9}$ \\
Cortical Index & $0.6 \pm 0.04$ & $0.62 \pm 0.04$ & $0.69 \pm 0.06$ & 0.66 & 0.302 \\
Canal Flare Index & $4.66 \pm 0.7$ & $4.84 \pm 0.64$ & $4.76 \pm 1.01$ & 5.4 & 0.426 \\
Canal to Calcar ratio & $0.62 \pm 0.1$ & $0.6 \pm 0.09$ & $0.62 \pm 0.09$ & 0.63 & 0.965 \\
\hline
\end{tabular}

All values are expressed as mean \pm standard deviation

COR center of rotation, $C C D$ angle centrum-collum-diaphyseal angle

Significant values in bold letters

\section{Discussion}

The results of this present study show minor increase in HO regardless of following or overruling the preoperative digital templating. Additionally, leg length difference was also without any statistically significant difference in both groups. Accurate reconstruction of hip geometry in THA is essential and has influence on clinical outcome, dislocation risk, range of motion, impingement, abductor muscle strength, and polyethylene wear [31-33]. The impact of offset reconstruction on the clinical outcome has been extensively examined. Innmann et al. [34] reported the best improvement in clinical outcome with a combination of complete to slightly increased $\mathrm{HO}( \pm 5 \mathrm{~mm})$ reconstruction and a marginal LLD in short stem THA with Fitmore ${ }^{\circledR}$ hip stem. Mahmood et al. [31] reported weaker hip abductor muscle strength in patients with a decrease in $\mathrm{HO}$ by more than $5 \mathrm{~mm}$. Sariali et al. [33] reported comparable findings with altered gait with asymmetry between both hips, reduced range of motion, and a lower maximal swing speed on the operated side for patients with a minimum decrease in $\mathrm{FO}$ of $15 \%$. Cassidy et al. [9] reported that a decrease in FO of more than $5 \mathrm{~mm}$ resulted in worse Western Ontario and McMaster Universities Arthritis Index (WOMAC) scores compared to patients with reconstructed or increased FO. Our results show an increase of HO in several groups compared to a healthy contralateral hip. However, a significant increase of $\mathrm{HO}$ was observed in cases of an overruling by one offset option higher than templated $(p=0.025)$. In all other groups $\mathrm{HO}$ was increased without significant difference to the contralateral healthy hip. An increase in $\mathrm{HO}$ of $\geq 5 \mathrm{~mm}$ compared to the contralateral normal hip negatively affects polyethylene wear [32]. We report values in a range under an increase of $5 \mathrm{~mm}$ for all groups, which is suggested to be superior for clinical functional outcome [34] and for polyethylene wear [32].
Overruling the preoperative digital templating did not pose a risk for leg length difference. Adequate reconstruction of HO and LL is considered as clinically important in THA $[22,34]$. However, the literature on leg length difference after THA and its clinical influence are inconsistent [34]. The consensus agreement recommends a LLD, that is kept to a minimum $[31,35]$. In all groups leg length was restored without significant differences independently of following or overruling preoperative templating.

Successful preoperative digital templating also relies on the correct X-ray and its quality. Merle et al.[25] described a risk of underestimation of FO in AP pelvis X-rays with an average of $13 \%$ compared to CT scans. In case of male patients, the effect could be additive because of averagely higher FO. Therefore, the preoperatively templated offset option and size might be too small because of the used X-ray. In this study, AP X-rays of the pelvis were used. Therefore, the use of a different offset option intraoperatively was necessary, because of an underestimation on the preoperative template.

Several limitations of the study have to be addressed. Firstly, we tried to minimize a potential selection bias with very strict inclusion criteria. We present a consecutive cohort with over 1000 THAs, which was reviewed for inclusion. Only patients with a single implant design and approach were included. A homogeneous study cohort was created by excluding patients with a bilateral hip disease (Kellgren Lawrence $>$ grade 1). Both study groups were tested for differences in age at surgery, BMI, laterality, and gender without any significance. Also, preoperative measurements were tested without any statistically significant differences. Furthermore, we aimed to increase reliability of the measurements and results by restricting inclusion based on preoperative diagnosis. We excluded all forms of secondary osteoarthritis of the hip and development dysplasia of the hip Crowe grade II to IV. Prior surgery before THA was also 
Table 4 Postoperative radiographic measurements and testing for statistically significant differences between the operated side and the contralateral healthy hip

\begin{tabular}{|c|c|c|c|}
\hline & THA & Healthy hip & $p$ value \\
\hline \multicolumn{4}{|l|}{ Hip offset (HO) (mm) } \\
\hline Offset 0 & $74 \pm 9$ & $71.9 \pm 7.8$ & 0.405 \\
\hline Offset +1 & $77.6 \pm 7$ & $73.4 \pm 5.9$ & 0.025 \\
\hline Offset -1 & $77.55 \pm 11.3$ & $74.7 \pm 10.5$ & 0.276 \\
\hline Offset -2 & 84 & 78 & - \\
\hline Offset $0 /$ Size 0 & $75.8 \pm 10.6$ & $72.9 \pm 8.7$ & 0.562 \\
\hline Offset $0 /$ Size $\geq+1$ & $75.3 \pm 13.2$ & $72.7 \pm 12.6$ & 0.575 \\
\hline Offset $0 /$ Size $\geq-1$ & $72.3 \pm 6.5$ & $70.9 \pm 6$ & 0.468 \\
\hline Offset \pm 1 option & $77.8 \pm 8.5$ & $74 \pm 7.5$ & 0.035 \\
\hline \multicolumn{4}{|c|}{ Acetabular Offset (AO) (mm) } \\
\hline Offset 0 & $29.3 \pm 3.3$ & $33.1 \pm 4$ & $<0.001$ \\
\hline Offset +1 & $28 \pm 4.1$ & $33.2 \pm 3.9$ & $<0.001$ \\
\hline Offset -1 & $30.6 \pm 3.5$ & $31.6 \pm 5.7$ & 0.408 \\
\hline Offset -2 & 31 & 31 & - \\
\hline Offset $0 /$ Size 0 & $29.1 \pm 2.8$ & $33.1 \pm 4.2$ & $<0.001$ \\
\hline Offset $0 /$ Size $\geq+1$ & $31 \pm 4.7$ & $35.3 \pm 6.3$ & 0.227 \\
\hline Offset $0 /$ Size $\geq-1$ & $29.2 \pm 3.3$ & $32.7 \pm 3.3$ & $<0.001$ \\
\hline Offset \pm 1 option & $29 \pm 4$ & $32.6 \pm 4.5$ & $<0.001$ \\
\hline \multicolumn{4}{|c|}{ Femoral Offset (FO) (mm) } \\
\hline Offset 0 & $44.7 \pm 8.5$ & $38.8 \pm 6$ & $<0.001$ \\
\hline Offset +1 & $49.6 \pm 6.3$ & $40.1 \pm 5.1$ & $<0.001$ \\
\hline Offset -1 & $46.9 \pm 9.5$ & $43.1 \pm 5.4$ & 0.167 \\
\hline Offset -2 & 53 & 47 & - \\
\hline Offset $0 /$ Size 0 & $46.7 \pm 10.2$ & $39.8 \pm 6.3$ & 0.014 \\
\hline Offset $0 /$ Size $\geq+1$ & $44.3 \pm 10.6$ & $37.3 \pm 6.5$ & 0.334 \\
\hline Offset $0 /$ Size $\geq-1$ & $43.2 \pm 6.4$ & $38.2 \pm 5.6$ & 0.003 \\
\hline Offset \pm 1 option & $48.8 \pm 7.4$ & $41.3 \pm 5.3$ & $<0.001$ \\
\hline \multicolumn{4}{|c|}{$\begin{array}{l}\text { Vertical position of the COR } \\
(\mathrm{mm})\end{array}$} \\
\hline Offset 0 & $19.1 \pm 3.4$ & $14 \pm 4$ & $<0.001$ \\
\hline Offset +1 & $20.9 \pm 5$ & $15.6 \pm 3.5$ & $<0.001$ \\
\hline Offset -1 & $16.8 \pm 3$ & $14.1 \pm 1.8$ & 0.021 \\
\hline Offset -2 & 15 & 12 & - \\
\hline Offset $0 /$ Size 0 & $19.2 \pm 2.6$ & $13.7 \pm 3.5$ & $<0.001$ \\
\hline Offset $0 /$ Size $\geq+1$ & $19.8 \pm 3.5$ & $15.2 \pm 3.8$ & 0.045 \\
\hline Offset $0 /$ Size $\geq-1$ & $18.9 \pm 4$ & $13.9 \pm 4.4$ & $<0.001$ \\
\hline Offset \pm 1 option & $19.3 \pm 4.7$ & $15 \pm 3.1$ & $<0.001$ \\
\hline \multicolumn{4}{|l|}{$\operatorname{LLD}(\mathrm{mm})$} \\
\hline Offset 0 & -0.3 & & 0.323 \\
\hline Offset +1 & -0.3 & & \\
\hline Offset -1 & -0.1 & & \\
\hline Offset -2 & 6 & & \\
\hline Offset $0 /$ Size 0 & $0.3 \pm 5.2$ & & 0.157 \\
\hline Offset $0 /$ Size $\geq+1$ & $0.7 \pm 3.6$ & & \\
\hline Offset $0 /$ Size $\geq-1$ & $-0.9 \pm 5.6$ & & \\
\hline Offset \pm 1 option & $0.0 \pm 5$ & & \\
\hline
\end{tabular}

excluded. However, mild hip dysplasia (lateral center-edge angle $20^{\circ}-25^{\circ}$ ), coxa profunda, and morphologic alterations related to cam- or pincer-type impingement were included, because these changes might be subtle and cannot be reliably identified in the present study cohort with end-stage disease. Therefore, we conclude that the findings in the present study are applicable for primary osteoarthritis and care must be taken when applying our findings on secondary osteoarthritis or high grades of development dysplasia of the hip. Secondly, we address the fact of taking measurements on plain radiographs. FO is underestimated by approximately $13 \%$ on plain radiographs [25]. Additionally, radiographic measurement of leg length difference does not necessarily reflect clinical leg length difference [36]. However, our measurements are easily reproducible, applicable in daily routine, and less invasive regarding radiation exposure. Furthermore, we postulate variances in inter- and intraobserver reliability in measuring clinical leg length difference. We acknowledge the restrictions of measurements on plain radiographs. But with implementing strict inclusion criteria and by using reproducible and well-described landmarks for measuring, we postulate a sufficient reduction of these limitations.

\section{Conclusion}

Intraoperative overruling of the preoperative digital template in cementless short stem total hip arthroplasty results in an increase of hip offset compared to a contralateral healthy hip. However, the increase is marginal and clearly under $5 \mathrm{~mm}$ compared to the contralateral healthy hip.

Acknowledgements Not applicable.

Authors contributions ML wrote the manuscript, performed the statistical analysis, designed the study, and contributed to acquisition of data and interpretation of the data. CS jointly conceived the study contributed to interpretation of the data. RH and TG revised the manuscript. JA was involved in the acquisition of data and interpretation of the data. AK jointly conceived the study, performed statistical analysis, edited the manuscript, and contributed to interpretation of the data.

Funding Open access funding provided by Johannes Kepler University Linz. This study was conducted without any funding or benefits from a commercial party.

Availability of data and materials Data and materials are available on request.

\section{Declarations}

Competing interests One co-author (R.H) has received consultant honoraria of ZimmerBiomet, Europe, outside the submitted work. The authors report personal fees paid to one co-author (T.G.) during the conduct of the study from Zimmer Biomet, Europe and from Depuy Synthes Orthopädie Gmbh, Peter Brehm GmbH, and ImplanTec GmbH 
outside the submitted work. The authors report research grants paid to our institution during the conduct of the study from Zimmer Biomet, Europe, Mathys AG Switzerland, and Anika Therapeutics outside the submitted work.

Ethical approval This study received ethical approval from the local institutional review board of the "Ethikkommission OO" of the Johannes Kepler University Linz (JKU Linz) (EK-No.: 1239/2019).

Consent to participate This study was approved by the institutional review board (EK-No.: 1239/2019) in accordance with the World Medical Association Declaration of Helsinki. Because of the retrospective evaluation of pre-existing medical records, an informed consent was not required.

Consent to publish Because of the retrospective evaluation of preexisting medical records, a consent to publish was not obtained.

Open Access This article is licensed under a Creative Commons Attribution 4.0 International License, which permits use, sharing, adaptation, distribution and reproduction in any medium or format, as long as you give appropriate credit to the original author(s) and the source, provide a link to the Creative Commons licence, and indicate if changes were made. The images or other third party material in this article are included in the article's Creative Commons licence, unless indicated otherwise in a credit line to the material. If material is not included in the article's Creative Commons licence and your intended use is not permitted by statutory regulation or exceeds the permitted use, you will need to obtain permission directly from the copyright holder. To view a copy of this licence, visit http://creativecommons.org/licenses/by/4.0/.

\section{References}

1. Kutzner KP, Kovacevic MP, Roeder C, Rehbein P, Pfeil J (2015) Reconstruction of femoro-acetabular offsets using a shortstem. Int Orthop 39(7):1269-1275. https://doi.org/10.1007/ s00264-014-2632-3

2. Gustke K (2012) Short stems for total hip arthroplasty: initial experience with the Fitmore stem. J Bone Jt Surg Br 94(11 Suppl A):47-51. https://doi.org/10.1302/0301-620X.94B11.30677

3. Pipino F, Molfetta L (1993) Femoral neck preservation in total hip replacement. Ital J Orthop Traumatol 19(1):5-12

4. Gkagkalis G, Goetti P, Mai S, Meinecke I, Helmy N, Bosson D, Kutzner KP (2019) Cementless short-stem total hip arthroplasty in the elderly patient - is it a safe option?: a prospective multicentre observational study. BMC Geriatr 19(1):112. https://doi.org/10. 1186/s12877-019-1123-1

5. Snijders TE, van Erp JHJ, de Gast A (2019) Restoring femoral offset and leg length; the potential of a short curved stem in total hip arthroplasty. J Orthop 16(5):396-399. https://doi.org/10.1016/j. jor.2019.04.013

6. Pisecky L, Hipmair G, Schauer B, Bohler N (2018) 30-years of experience with the cementless implanted Alloclassic total hip arthroplasty system - an ultra-long-term follow-up. J Orthop 15(1):18-23. https://doi.org/10.1016/j.jor.2017.11.003

7. McGrory BJ, Morrey BF, Cahalan TD, An KN, Cabanela ME (1995) Effect of femoral offset on range of motion and abductor muscle strength after total hip arthroplasty. J Bone Joint Surg Br 77(6):865-869

8. Asayama I, Chamnongkich S, Simpson KJ, Kinsey TL, Mahoney OM (2005) Reconstructed hip joint position and abductor muscle strength after total hip arthroplasty. J Arthroplasty 20(4):414-420. https://doi.org/10.1016/j.arth.2004.01.016

9. Cassidy KA, Noticewala MS, Macaulay W, Lee JH, Geller JA (2012) Effect of femoral offset on pain and function after total hip arthroplasty. J Arthroplasty 27(10):1863-1869. https://doi.org/10. 1016/j.arth.2012.05.001

10. Shemesh SS, Robinson J, Keswani A, Bronson MJ, Moucha CS, Chen D (2017) The accuracy of digital templating for primary total hip arthroplasty: is there a difference between direct anterior and posterior approaches? J Arthroplasty 32(6):1884-1889. https://doi.org/10.1016/j.arth.2016.12.032

11. Bono JV (2004) Digital templating in total hip arthroplasty. J Bone Jt Surg Am 86-A(Suppl 2):118-122. https://doi.org/10.2106/ 00004623-200412002-00016

12. Kamada S, Naito M, Nakamura Y, Kiyama T (2011) Hip abductor muscle strength after total hip arthroplasty with short stems. Arch Orthop Trauma Surg 131(12):1723-1729. https://doi.org/10.1007/ s00402-011-1350-1

13. Eggli S, Pisan M, Muller ME (1998) The value of preoperative planning for total hip arthroplasty. J Bone Jt Surg Br 80(3):382390. https://doi.org/10.1302/0301-620x.80b3.7764

14. Heinert G, Hendricks J, Loeffler MD (2009) Digital templating in hip replacement with and without radiological markers. J Bone Jt Surg Br 91(4):459-462. https://doi.org/10.1302/0301-620X.91B4. 21730

15. Kim YH, Kim VE (1993) Uncemented porous-coated anatomic total hip replacement. Results at six years in a consecutive series. J Bone Jt Surg Br 75(1):6-13

16. Schmidutz F, Steinbruck A, Wanke-Jellinek L, Pietschmann M, Jansson V, Fottner A (2012) The accuracy of digital templating: a comparison of short-stem total hip arthroplasty and conventional total hip arthroplasty. Int Orthop 36(9):1767-1772. https://doi. org/10.1007/s00264-012-1532-7

17. Jung S, Neuerburg C, Kappe T, Wernerus D, Reichel H, Bieger R (2012) Validity of digital templating in total hip arthroplasty: impact of stem design and planner's experience. Z Orthop Unfall 150(4):404-408. https://doi.org/10.1055/s-0031-1298386

18. Brenneis M, Braun S, van Drongelen S, Fey B, Tarhan T, Stief F, Meurer A (2020) Accuracy of preoperative templating in total hip arthroplasty with special focus on stem morphology: a randomized comparison between common digital and three-dimensional planning using biplanar radiographs. J Arthroplasty. https:// doi.org/10.1016/j.arth.2020.10.016

19. Shaarani SR, McHugh G, Collins DA (2013) Accuracy of digital preoperative templating in 100 consecutive uncemented total hip arthroplasties: a single surgeon series. J Arthroplasty 28(2):331337. https://doi.org/10.1016/j.arth.2012.06.009

20. Crowe JF, Mani VJ, Ranawat CS (1979) Total hip replacement in congenital dislocation and dysplasia of the hip. J Bone Joint Surg Am 61(1):15-23

21. Kellgren JH, Lawrence JS (1957) Radiological assessment of osteo-arthrosis. Ann Rheum Dis 16(4):494-502. https://doi.org/ 10.1136/ard.16.4.494

22. Dastane M, Dorr LD, Tarwala R, Wan Z (2011) Hip offset in total hip arthroplasty: quantitative measurement with navigation. Clin Orthop Relat Res 469(2):429-436. https://doi.org/10.1007/ s11999-010-1554-7

23. Innmann MM, Streit MR, Kolb J, Heiland J, Parsch D, Aldinger PR, Konigshausen M, Gotterbarm T, Merle C (2015) Influence of surgical approach on component positioning in primary total hip arthroplasty. BMC Musculoskelet Disord 16:180. https://doi.org/ 10.1186/s12891-015-0623-1

24. Wedemeyer C, Quitmann H, Xu J, Heep H, von Knoch M, Saxler G (2008) Digital templating in total hip arthroplasty with the Mayo stem. Arch Orthop Trauma Surg 128(10):1023-1029. https://doi.org/10.1007/s00402-007-0494-5 
25. Merle C, Waldstein W, Pegg E, Streit MR, Gotterbarm T, Aldinger PR, Murray DW, Gill HS (2012) Femoral offset is underestimated on anteroposterior radiographs of the pelvis but accurately assessed on anteroposterior radiographs of the hip. J Bone Joint Surg Br 94(4):477-482. https://doi.org/10.1302/0301-620X.94B4. 28067

26. Innmann MM, Spier K, Streit MR, Aldinger PR, Bruckner T, Gotterbarm T, Merle C (2018) Comparative analysis of the reconstruction of individual hip anatomy using 3 different cementless stem designs in patients with primary hip osteoarthritis. J Arthroplasty 33(4):1126-1132. https://doi.org/10.1016/j.arth.2017.11. 026

27. Waldt S, Eiber M, Wörtler K (2011) Messverfahren und Klassifikationen in der muskuloskelettalen Radiologie. Thieme, Stuttgart

28. Dorr LD, Faugere MC, Mackel AM, Gruen TA, Bognar B, Malluche HH (1993) Structural and cellular assessment of bone quality of proximal femur. Bone 14(3):231-242. https://doi.org/10. 1016/8756-3282(93)90146-2

29. Noble PC, Alexander JW, Lindahl LJ, Yew DT, Granberry WM, Tullos HS (1988) The anatomic basis of femoral component design. Clin Orthop Relat Res 235:148-165

30. Hoenig JM, Heisey DM (2001) The abuse of power. Am Stat 55(1):19-24. https://doi.org/10.1198/000313001300339897

31. Mahmood SS, Mukka SS, Crnalic S, Wretenberg P, Sayed-Noor AS (2016) Association between changes in global femoral offset after total hip arthroplasty and function, quality of life, and abductor muscle strength. A prospective cohort study of 222 patients. Acta Orthop 87(1):36-41. https://doi.org/10.3109/17453674. 2015.1091955
32. Little NJ, Busch CA, Gallagher JA, Rorabeck CH, Bourne RB (2009) Acetabular polyethylene wear and acetabular inclination and femoral offset. Clin Orthop Relat Res 467(11):2895-2900. https://doi.org/10.1007/s11999-009-0845-3

33. Sariali E, Klouche S, Mouttet A, Pascal-Moussellard H (2014) The effect of femoral offset modification on gait after total hip arthroplasty. Acta Orthop 85(2):123-127. https://doi.org/10.3109/ 17453674.2014.889980

34. Innmann MM, Maier MW, Streit MR, Grammatopoulos G, Bruckner T, Gotterbarm T, Merle C (2018) Additive influence of hip offset and leg length reconstruction on postoperative improvement in clinical outcome after total hip arthroplasty. J Arthroplasty 33(1):156-161. https://doi.org/10.1016/j.arth.2017.08.007

35. Whitehouse MR, Stefanovich-Lawbuary NS, Brunton LR, Blom AW (2013) The impact of leg length discrepancy on patient satisfaction and functional outcome following total hip arthroplasty. J Arthroplasty 28(8):1408-1414. https://doi.org/10.1016/j.arth. 2012.12.009

36. Tipton SC, Sutherland JK, Schwarzkopf R (2016) The assessment of limb length discrepancy before total hip arthroplasty. J Arthroplasty 31(4):888-892. https://doi.org/10.1016/j.arth.2015.10.026

Publisher's Note Springer Nature remains neutral with regard to jurisdictional claims in published maps and institutional affiliations. 